\title{
Principal Functions for High Powers of Operators
}

\author{
Dedicated to Professor Seiji Watanabe on his sixtieth birthday \\ Muneo CHŌ, Tadasi HURUYA, An Hyun KIM and Chunji LI \\ Kanagawa University, Niigata University, Changwon National University \\ and Northeastern University \\ (Communicated by K. Taniyama)
}

\begin{abstract}
For an operator $T$ with some trace class condition, let $g_{T^{n}}$ and $g_{T^{n}}^{P}$ be the principal functions related to the Cartesian decomposition $T^{n}=X_{n}+i Y_{n}$ and the polar decomposition $T^{n}=U_{n}\left|T^{n}\right|$ for a positive integer $n$, respectively. In this paper, we study properties of $g_{T^{n}}$ and $g_{T^{n}}^{P}$ and invariant subspaces of $T^{n}$.
\end{abstract}

\section{Introduction}

An operator below means a bounded linear operator on a separable infinite dimensional Hilbert space $\mathcal{H}$. The commutator of two operators $A, B$ is denoted by $[A, B]=A B-B A$. Let $\mathcal{C}_{1}$ be the set of trace-class operators of $B(\mathcal{H})$. Let $T$ be an operator such that $\left[T^{*}, T\right] \in$ $\mathcal{C}_{1}$. Pincus introduced the principal function $g_{T}$ related to the Cartesian decomposition $T=$ $X+i Y$. Properties of the principal function $g_{T}$ have been studied ([3], [6], [7], [9], [10]). Especially, C.A. Berger gave the principal functions $g_{T^{n}}$ of powers $T^{n}$ of $T$ in terms of $g_{T}$ and proved that for a sufficiently high $n, T^{n}$ has a non-trivial invariant subspace for a hyponormal operator $T$ ([1]). On the other hand, we have another principal function $g_{T}^{P}$ related to the polar decomposition $T=U|T|$ such that [|T|,U] $\in \mathcal{C}_{1}$ ([3], [4], [10]).

In this paper, we study properties of $g_{T^{n}}$ and $g_{T^{n}}^{P}$ and invariant subspaces of $T^{n}$.

\section{Theorem}

$\phi(r, z)$ is called Laurent polynomial if there exist a non-negative integer $N$ and polynomials $p_{k}(r)$ such that $\phi(r, z)=\sum_{k=-N}^{N} p_{k}(r) z^{k}$. For differentiable functions $P, Q$ of two variables $(x, y)$, let $J(P, Q)(x, y)=P_{x}(x, y) \cdot Q_{y}(x, y)-P_{y}(x, y) \cdot Q_{x}(x, y)$.

For an operator $T=X+i Y=U|T|$, we consider the following trace formulae:

Received October 4, 2004; revised February 11, 2005

2000 Mathematics Subject Classification. 47B20, 47A10.

Key words and phrases. Hilbert space, trace, principal function.

This research is partially supported by Grant-in-Aid Scientific Research No. 17540176. 


$$
\operatorname{Tr}([P(X, Y), Q(X, Y)])=\frac{1}{2 \pi i} \iint J(P, Q)(x, y) g_{T}(x, y) d x d y,
$$

for polynomials $P$ and $Q$.

(2) $\quad \operatorname{Tr}([\phi(|T|, U), \psi(|T|, U)])=\frac{1}{2 \pi} \iint J(\phi, \psi)\left(r, e^{i \theta}\right) e^{i \theta} g_{T}^{P}\left(e^{i \theta}, r\right) d r d \theta$

for Laurent polynomials $\phi$ and $\psi$.

If formula (1) holds, the function $g_{T}$ is called the principal function related to the Cartesian decomposition $T=X+i Y$. If formula (2) holds, the function $g_{T}^{P}$ is called the principal function related to the polar decomposition $T=U|T|$. For invertible operator $T$ such that $\left[T^{*}, T\right] \in \mathcal{C}_{1}$, there exist both $g_{T}$ and $g_{T}^{P}$ ([3], [5]).

First we start with the following

THEOREM 1. Let $T=X+i Y=U|T|$ be an operator satisfying the following trace formula:

$$
\operatorname{Tr}([\phi(|T|, U), \psi(|T|, U)])=\frac{1}{2 \pi} \iint J(\phi, \psi)\left(r, e^{i \theta}\right) e^{i \theta} g_{T}^{P}\left(e^{i \theta}, r\right) d r d \theta
$$

for any Laurent polynomials $\phi$ and $\psi$. Then the principal function $g_{T}(x, y)$ related to the Cartesian decomposition $T=X+i Y$ of $T$ exists and it is given by $g_{T}(x, y)=g_{T}^{P}\left(e^{i \theta}, r\right)$, where $x+i y=r e^{i \theta}$.

Proof. Let $P$ and $Q$ be polynomials of two variables $(x, y)$. First note that

$$
\operatorname{Tr}([P(X, Y), Q(X, Y)])=\operatorname{Tr}\left(\left[P\left(\frac{T+T^{*}}{2}, \frac{T-T^{*}}{2 i}\right), Q\left(\frac{T+T^{*}}{2}, \frac{T-T^{*}}{2 i}\right)\right]\right) .
$$

Put

$$
\tilde{P}(r, z)=P\left(\frac{z r+r / z}{2}, \frac{r z-r / z}{2 i}\right) \text { and } \tilde{Q}(r, z)=Q\left(\frac{z r+r / z}{2}, \frac{r z-r / z}{2 i}\right) .
$$

Then both $\tilde{P}$ and $\tilde{Q}$ are Laurent polynomials and also the following equations hold:

$$
\begin{aligned}
& \tilde{P}_{r}(r, z)=P_{x}(r, z) \frac{z+1 / z}{2}+P_{y}(r, z) \frac{z-1 / z}{2 i}, \\
& \tilde{P}_{z}(r, z)=\frac{r}{2} P_{x}(r, z)\left(1-\frac{1}{z^{2}}\right)+\frac{r}{2 i} P_{y}(r, z)\left(1+\frac{1}{z^{2}}\right), \\
& \tilde{Q}_{r}(r, z)=Q_{x}(r, z) \frac{z+1 / z}{2}+Q_{y}(r, z) \frac{z-1 / z}{2 i}, \\
& \tilde{Q}_{z}(r, z)=\frac{r}{2} Q_{x}(r, z)\left(1-\frac{1}{z^{2}}\right)+\frac{r}{2 i} Q_{y}(r, z)\left(1+\frac{1}{z^{2}}\right) .
\end{aligned}
$$


Hence we obtain

$$
J(\tilde{P}, \tilde{Q})(r, z)=J(P, Q)(x, y) \frac{r}{z i}
$$

Therefore, it holds

$$
J(\tilde{P}, \tilde{Q})\left(r, e^{i \theta}\right)=J(P, Q)(x, y) \frac{r}{i e^{i \theta}}
$$

Since $P(X, Y)=\tilde{P}(|T|, U)$ and $Q(X, Y)=\tilde{Q}(|T|, U)$, it holds

$$
\operatorname{Tr}([P(X, Y), Q(X, Y)])=\operatorname{Tr}([\tilde{P}(|T|, U), \tilde{Q}(|T|, U)]) .
$$

By (3) and (4), we have

$$
\begin{aligned}
\operatorname{Tr}([P(X, Y), Q(X, Y)]) & =\operatorname{Tr}([\tilde{P}(|T|, U), \tilde{Q}(|T|, U)]) \\
& =\frac{1}{2 \pi} \iint J(\tilde{P}, \tilde{Q})\left(r, e^{i \theta}\right) e^{i \theta} g_{T}^{P}\left(e^{i \theta}, r\right) d r d \theta \\
& =\frac{1}{2 \pi i} \iint J(P, Q)(x, y) g_{T}^{P}\left(e^{i \theta}, r\right) r d r d \theta
\end{aligned}
$$

Put $g_{T}(x, y)=g_{T}^{P}\left(e^{i \theta}, r\right)$ for $x+i y=r e^{i \theta}$. Using the transformation $x=r \cos \theta$ and $y=r \sin \theta$, we have

$$
\begin{aligned}
\frac{1}{2 \pi i} \iint J(P, Q)(x, y) g_{T}^{P}\left(e^{i \theta}, r\right) r d r d \theta & =\frac{1}{2 \pi i} \iint J(P, Q)(x, y) g_{T}(x, y) d x d y \\
& =\operatorname{Tr}([P(X, Y), Q(X, Y)])
\end{aligned}
$$

Since $P$ and $Q$ are arbitrary, by (1) it completes the proof.

If an operator $T=U|T|$ is invertible, then $U$ is unitary and [|T|,U] $\in \mathcal{C}_{1}$ implies $\left[T^{*}, T\right] \in \mathcal{C}_{1}$, because $\left[T^{*}, T\right]=|T|[|T|, U] U^{*}+[|T|, U]|T| U^{*}$. And equation (2) holds by [5, Theorem 4]. So we have the following

COROLlaRY 2. If an invertible operator $T=X+i Y=U|T|$ satisfies $[|T|, U] \in \mathcal{C}_{1}$, then $g_{T}(x, y)=g_{T}^{P}\left(e^{i \theta}, r\right)$, where $x+i y=r e^{i \theta}$.

For a relation between $g_{T}^{P}$ and $g_{T^{n}}^{P}$, we need the following Berger's result:

THEOREM 3 (Berger, Th.4[1]). For an operator $T$, if $\left[T^{*}, T\right] \in \mathcal{C}_{1}$, then for a positive integer $n$,

$$
g_{T^{n}}(x, y)=\sum_{(u+i v)^{n}=x+i y} g_{T}(u, v) .
$$

THEOREM 4. For an operator $T$ with $\left[T^{*}, T\right] \in \mathcal{C}_{1}$, if $\iint g_{T}(x, y) d x d y \neq 0$, then

$$
\lim _{n \rightarrow \infty} \operatorname{ess} \sup \left|g_{T^{n}}\right|=\infty \text {. }
$$


Proof. We choose a positive number $a$ such that $\|a T\|<1$. It holds $g_{a T}(x, y)=$ $g_{T}(x / a, y / a)($ cf. $[9$, p. 242,1)]). Hence we have

$$
\text { ess } \sup \left|g_{a T}(x, y)\right|=\operatorname{ess} \sup \left|g_{T}(x, y)\right| \text {. }
$$

Therefore, we may assume that $\|T\|<1$. Put $g(x+i y)=g_{T}(x, y)$ and $g_{n}(x+i y)=$ $g_{T^{n}}(x, y)$. Let $m_{2}$ be the planar Lebesgue measure. Since $m_{2}(\{0\})=0$, we consider in the set $\mathbf{C}-\{0\}$. Put $S(n, k)=\left\{z \in \mathbf{C}-\{0\}: \frac{2 \pi(k-1)}{n} \leq \arg z<\frac{2 \pi k}{n}\right\} \quad(k=1, \cdots, n)$. Then by Theorem 3 we have

$$
g_{n}\left(r^{n} e^{i \theta}\right)=\sum_{k=1}^{n} g\left(r e^{i(\theta+2 \pi(k-1)) / n}\right)
$$

Hence it holds

$$
\begin{aligned}
\iint g_{n}\left(r^{n} e^{i \theta}\right) r d \theta d r & =\sum_{k=1}^{n} \iint g\left(r e^{i(\theta+2 \pi(k-1)) / n}\right) r d \theta d r \\
& =\sum_{k=1}^{n} n \iint_{S(n, k)} g\left(r e^{i \theta}\right) r d \theta d r=n \iint g\left(r e^{i \theta}\right) r d \theta d r \\
& =n \iint g_{T}(x, y) d x d y \neq 0
\end{aligned}
$$

for every $n$. By [2, Theorem 3.3], for an operator $S$ with $\left[S^{*}, S\right] \in \mathcal{C}_{1}$, the support of $g_{S}$ is contained in $[-\|S\|,\|S\|] \times[-\|S\|,\|S\|]$. Let $A_{n}$ denote the support of $g_{T^{n}}$. Since $\|T\|<1$, it holds $\lim _{n \rightarrow \infty} m_{2}\left(A_{n}\right)=0$. Hence

$$
\lim _{n \rightarrow \infty} \text { ess sup }\left|g_{T^{n}}\right|=\lim _{n \rightarrow \infty} \text { ess sup }\left|g_{n}\right|=\infty \text {. }
$$

We remark that a hyponormal operator $T$ with $0 \neq\left[T^{*}, T\right] \in \mathcal{C}_{1}$ satisfies $\iint g_{T}(x, y) d x d y \neq 0$.

Applying Corollary 2 and Theorem 4 to $T^{n}$, we have the following.

COROLlary 5. For an operator $T=U|T|$, let $T^{n}=U_{n}\left|T^{n}\right|$ be the polar decomposition of $T^{n}(n=1,2, \cdots)$. If $\left[\left|T^{n}\right|, U_{n}\right] \in \mathcal{C}_{1}$ for every non-negative integer $n$ and $\iint g_{T}^{P}\left(e^{i \theta}, r\right) r d \theta d r \neq 0$, then

$$
\lim _{n \rightarrow \infty} \operatorname{ess} \sup \left|g_{T^{n}}^{P}\right|=\infty
$$

Let $T=U|T|$ and $T^{n}=U_{n}\left|T^{n}\right|$ be the polar decompositions of $T$ and $T^{n}$, respectively. Then it holds that if $\left[T^{*}, T\right] \in \mathcal{C}_{1}$, then $\left[T^{* n}, T^{n}\right] \in \mathcal{C}_{1}$ for any positive integer $n$. On the other hand, in the polar decomposition case, it is not clear whether $[|T|, U] \in \mathcal{C}_{1}$ impilies $\left[\left|T^{n}\right|, U_{n}\right] \in \mathcal{C}_{1}$ even if $n=2$. If $T$ is invertible and $[|T|, U] \in \mathcal{C}_{1}$, then, for every $n$, it holds $\left[\left|T^{n}\right|, U_{n}\right] \in \mathcal{C}_{1}$ by $[5$, Theorem 3$]$. 
Next we consider operators with cyclic vectors. First we need the following result. We remark that the proof of [9, Theorem X.4.3] works still for a pair of operators with trace class self-commutator.

Theorem 6 (Martin and Putinar, Th.X.4.3 [9]). Let $g_{T}$ and $g_{V}$ be the principal functions of operators $T$ and $V$ such that $\left[T^{*}, T\right],\left[V^{*}, V\right] \in \mathcal{C}_{1}$, respectively. If there exists an operator $A \in \mathcal{C}_{1}$ such that $A V=T A$ and $\operatorname{ker}(A)=\operatorname{ker}\left(A^{*}\right)=\{0\}$, then $g_{T} \leq g_{V}$.

Proof of the following lemma is based on it of [9, Corollary X.4.4].

LEMmA 7. Let $T$ be an operator such that $\left[T^{*}, T\right] \in \mathcal{C}_{1}$ and $\sigma(T)$ is an infinite set. If $T$ has a cyclic vector, then $g_{T} \leq 1$.

Proof. We may assume that $\|T\|<1$. Let $\xi$ be a cyclic vector for $T$. Define an operator $A: \ell^{2}(\mathbf{N}) \rightarrow \mathcal{H}$ by

$$
A e_{k}=T^{k} \xi, \quad k \geq 0,
$$

where $\left\{e_{k}\right\}$ denotes the standard basis of $\ell^{2}$. Let $V$ be the unilateral shift on $\ell^{2}(\mathbf{N})$. Then it holds $A V=T A$. It is easy to see that $\operatorname{ker}\left(A^{*}\right)=\{0\}$. Also we show $\operatorname{ker}(A)=\{0\}$. Assume that $\operatorname{ker}(A) \neq\{0\}$. Since $\operatorname{ker}(A) \neq\{0\}$ if and only if there exists a non-zero analytic function $f$ in the unit disk such that $f(T)=0$, we have $\{0\}=\sigma(f(T))=f(\sigma(T))$. Since $\sigma(T)$ has a limit point in the unit disk, we have $f=0$. It's a contradiction. Hence, we have $\operatorname{ker}(A)=\{0\}$.

By the similar way to Corollary X.4.4 of [9], it follows that $A$ is a trace class operator. Since $g_{V} \leq 1$, by Theorem 6 , we have

$$
g_{T} \leq g_{V} \leq 1 .
$$

Let $S$ be an operator having the principal function $g_{S}$ related to the Cartesian decomposition $S=X+i Y$. Then $g_{S^{*}}(x, y)=-g_{S}(x,-y)$. Hence, as a corollary of Lemma 7, we have the following.

Lemma 8. Let $T$ be an operator such that $\left[T^{*}, T\right] \in \mathcal{C}_{1}$ and $\sigma(T)$ is an infinite set. If $T^{*}$ has a cyclic vector, then $-1 \leq g_{T}$.

Finally, we give an invariant subspace result. Using a property of $g_{T^{n}}$, Berger showed that $(T-a I)^{n}$ has a non-trivial invariant subspace for any number $a$ satisfying $g_{T}(a) \neq 0$ and a sufficiently high $n$ (see also [8]).

We remark that if $K$ is a non-trivial invariant subspace for $S^{*}$, then $K^{\perp}$ is an invariant subspace for $S$.

THEOREM 9. Let $T$ be an operator such that $\left[T^{*}, T\right] \in \mathcal{C}_{1}$ and $\sigma(T)$ is an infinite set. Moreover, if $\iint g_{T}(x, y) d x d y \neq 0$, then, for a sufficiently high $n, T^{n}$ has a non-trivial invariant subspace.

Proof. For a positive integer $n$, it holds that $\left[T^{n *}, T^{n}\right] \in \mathcal{C}_{1}, \sigma\left(T^{n}\right)$ is an infinite set and $g_{T} \neq 0$. If both $T^{n}$ and $T^{* n}$ have cyclic vectors, then, from Lemmas 7 and 8 , 
$\left|g_{T^{n}}\right| \leq 1$. Hence from Theorem 4, for a sufficiently high $n, T^{n}$ or $T^{* n}$ has a non-trivial invariant subspace. If $T^{* n}$ has a non-trivial invariant subspace, so does $T^{n}$. This completes the proof.

\section{References}

[ 1 ] C. A. BERGER, Sufficiently high powers of hyponormal operators have rationally invariant subspaces, Integr Equat. Oper. Th. 1/3 (1978), 444-447.

[ 2 ] R. W. CAREY and J. D. Pincus, Almost commuting algebras, Lecture Notes in Math., 574, Springer-Verlag, Berlin (1973), 19-43.

[ 3 ] R. W. CAREY and J. D. Pincus, Mosaics, principal functions, and mean motion in von-Neumann algebras, Acta Math. 138 (1977), 153-218.

[ 4 ] M. Chō and T. HuruYA, Trace formulae of $p$-hyponormal operators, Studia Math. 161 (2004), 1-18.

[ 5 ] M. Chō, T. HuruYA and C. LI, Trace formulae associated with the polar decomposition of operators, Math. Proc. Royal Irish Acad. 105A (2005), 57-69.

[ 6 ] K. F. Clancey, Seminormal operators, Lecture Notes in Math., 742, Springer-Verlag, Berlin-HeidelbergNew York (1979).

[ 7 ] J. W. Helton and R. Howe, Integral operators, commutator traces, index and homology, Proceedings of a conference on operator theory, Lecture Notes in Math., 345, Springer-Verlag, Berlin-Heidelberg-New York (1973).

[ 8 ] I. B. Jung, E. Ko and C. PeArCy, Aluthge transforms of operators, Integr. Equat. Oper. Th. 37 (2000), 437-448.

[9] M. MARTIN and M. PUTINAR, Lectures on hyponormal operators, Birkhäuser Verlag, Basel (1989).

[10] D. XIA, Spectral theory of hyponormal operators, Birkhäuser Verlag, Basel (1983).

Present Addresses:

MUNEO CHŌ

DEPARTMENT OF MATHEMATICS KANAGAWA UNIVERSITY,

YOKOHAMA, 221-8686 JAPAN.

e-mail: chiyom01@ kanagawa-u.ac.jp

TADASI HURUYA

FACUlTy of EduCATION AND Human SCIENCES, NiIgATA UNIVERSity,

NIIGATA, 950-2181 JAPAN.

e-mail: huruya@ed.niigata-u.ac.jp

AN HYUN KIM

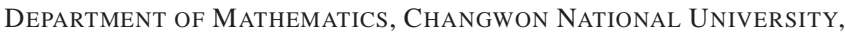

KYONGNAM, 641-773 KOREA.

e-mail: ahkim@changwon.ac.kr

CHUNJI Li

Institute of System Science, College of Sciences, Northeastern University, SHENYANG, LiAONING, 110-004 P. R. ChInA.

e-mail: chunjili@hanmail.net 